\title{
Satisfacción del cliente: Estudio desde las metodologías de calidad. Caso Black Mouth
}

\author{
Pablo Alfredo Rituay Trujillo ${ }^{1}$ \\ Adriano Rogerio Bruno Tech ${ }^{2}$ \\ Renata Lima Zuccherelli de Oliveira ${ }^{3}$ \\ Jonathan Alberto Campos Trigoso ${ }^{4}$
}

\section{Resumen}

Para atender las exigencias y necesidades del consumidor de café en taza y ofrecer un producto de calidad se aplicó el método de despliegue de la función de calidad (Quality Function Deployment - QFD) en la cafetería Black Mouth de la ciudad de Chachapoyas, con el objetivo principal de determinar el nivel de satisfacción del cliente. Se aplicaron 183 encuestas a consumidores de la cafetería durante el año 2019, se identificaron las necesidades y expectativas (Voz del Cliente - VoC) de los clientes. La evaluación de la satisfacción del cliente en la escala de Likert fue de buena y óptima con un $25.1 \%$ y $52.7 \%$ respectivamente, al mismo tiempo se evaluaron competitivamente cada uno de los productos (café cappuccino, café americano y frappe). El producto que presentó un menor desempeño fue el Frappe. En seguida se definió las características de calidad de éste a través de la casa de la calidad (House of Quality - HOQ), de esta manera se

\section{Recibido: 15-05-19 Aceptado: 17-09-19}

1 Ingeniero en Agronegocios, Instituto de Investigación en Negocios Agropecuarios, Facultad de Ingeniería Zootecnista, Agronegocios y Biotecnología de la Universidad Nacional Toribio Rodríguez de Mendoza de Amazonas. E-mail: pablo.rituay@untrm.edu.pe. Orcid: https:// orcid.org/0000-0003-4338-6057

2 Doctor en Zootecnia. Docente de la Facultad de Zootecnia e Ingeniería de Alimentos de la Universidad de São Paulo, Brasil. Rua Duque de Caxias Norte 225, Jardin Elite - CEP 13635900 - Pirassununga (São Paulo), Brasil. Email: adriano.tech@usp.br.Orcid: https:// orcid.org/0000-0003-2628-1915

3 Máster en Gestión e innovación de la Industria Animal, Facultad de Zootecnia e Ingeniería de Alimentos de la Universidad de São Paulo, Brasil. Rua Duque de Caxias Norte 225, Jardin Elite - CEP 13635900 - Pirassununga (São Paulo), Brasil. Email: rezuc@usp.br. Orcid: https://orcid.org/0000-0002-2786-9350

$4 \quad$ Máster en Economía Agroalimentaria y del Medio Ambiente,Instituto de Investigación en Negocios Agropecuarios, Facultad de Ingeniería Zootecnista, Agronegocios y Biotecnología de la Universidad Nacional Toribio Rodríguez de Mendoza de Amazonas. E-mail: jonathan. campos@untrm.edu.pe. Orcid: https://orcid.org/0000-0002-4605-6005 
logró visualizar aquellas características en las que se debería concentrar los esfuerzos futuros para un mejor posicionamiento competitivo del mismo en los consumidores de la cafetería.

Palabras clave: despliegue de la función de calidad; calidad; cafetería; cliente

\title{
Customer satisfaction: Study from quality methodologies. Black Mouth case
}

\begin{abstract}
In order to meet the demands and needs of the coffee-cup consumer and offer a quality product, the Quality Function Deployment (QFD) deployment method was applied in the Black Mouth cafeteria of the city of Chachapoyas, with the objective Main to determine the level of customer satisfaction, for which 183 surveys were applied to consumers of the cafeteria during the year 2019, the needs and expectations (Customer Voice - VoC) of the customers were identified. The evaluation of customer satisfaction on the Likert scale was good and optimal with $25.1 \%$ and $52.7 \%$ respectively, at the same time each product was competitively evaluated (cappuccino coffee, American coffee and frappe). The product that presented the lowest performance was the Frappe. Then the quality characteristics of the latter were defined through the House of Quality (HOQ), in this way it was possible to visualize those characteristics in which future efforts should be concentrated for a better competitive positioning of the same in the cafeteria consumers.
\end{abstract}

Keywords: Deployment of the quality function; Quality; Cafeteria; Client.

\section{Introducción}

En la actualidad, los clientes son cada vez más exigentes en cuanto a la calidad de los servicios recibidos, producto del dinamismo de los mercados, la competitividad y la globalización, como respuesta a ello y con el objetivo de satisfacer sus exigencias y demandas, y asegurar ventaja competitiva y la sostenibilidad de las organizaciones es necesaria la evaluación de la satisfacción de los clientes y su lealtad (Evans \& Lindsay, 2015; Mejías \& Manrique 2011).

Tavira \& Estrada (2015), afirman que la evaluación de la satisfacción de los clientes debe ser un objetivo primordial en cualquier organización de servicio y el desarrollo de indicadores de la calidad; es una forma adecuada para diagnosticar el desempeño de un proceso, de esta manera será posible priorizar aquellos puntos críticos para satisfacer las necesidades y expectativas de los clientes.

Las organizaciones entonces deben contar con productos y servicios de alta calidad para satisfacer a sus clientes, siendo de fundamental importancia proporcionar a directivos 
metodologías y herramientas de análisis que permitan identificar las percepciones de calidad positivas y negativas del cliente y traducirlas en características de nuevos productos o fortalecer las bondades de los ya existentes (Gómez \& Suárez, 2005; Abdul \& Berawi 2002).

El despliegue de la función de calidad (QFD, por sus siglas en inglés), desarrollada por el investigador japonés Dr. Yoji Akao en 1960, permite la planificación efectiva para el desarrollo de productos, en la cual la voz vagamente expresada del cliente se transforma en ingeniería y características para un producto o servicio, que puede mejorar la capacidad de ingresos de la organización, así como reducir el tiempo de diseño y costos asociados al mismo (Zhang et al, 2019; Cheng, 2007).

El consumo mundial de café sigue una tendencia de crecimiento; para la campaña 2017/18 se estima que habría alcanzado un volumen récord de 158,5 millones de sacos concentrándose en cafés comunes, alrededor del $85 \%$ y $90 \%$ del consumo mundial, el $15 \%$ o $10 \%$ restante se orienta a los mercados certificados o sostenibles y los especiales, aquellos que son comercializados en cafeterías especializadas (Proyecto Café y Clima, 2017)

En este contexto, el objetivo general de la investigación fue determinar el nivel de satisfacción del cliente de la cafetería Black Mouth en la ciudad de Chachapoyas, para ello se aplicaron 183 encuestas a consumidores. A partir de los resultados producto del procesamiento $\mathrm{y}$ análisis de las encuestas se usó la metodología del despliegue de la función de calidad, para transformar la voz del cliente y determinar de forma efectiva aquellos aspectos que deben ser priorizados para mejorar las características de calidad.

\section{Despliegue de la Función de Calidad}

En la investigación se utilizó la metodología del despliegue de la función de calidad (QFD), desarrollada a través del uso de la matriz denominada casa de la calidad (House of Quality - HOQ), la aplicación de ésta resulta de importancia fundamental y estratégica para trasladar aquello que necesita y busca el cliente a requerimientos de calidad internos de la organización (Moubachir \& Bouami, 2015; Kazemzadeh et al, 2009; Lorenzo et al, 2004)

La casa de la calidad permite sistematizar los requerimientos del cliente y las características técnicas del producto, plasmando la información en una misma figura, junto a otras variables que facilitan el diseño de un producto, la matriz $\mathrm{HOQ}$ se usa a menudo para identificar las relaciones entre requisitos facilitando la comunicación de a los miembros del equipo alcanzando una representación formal de las exigencias de los clientes o consumidores para el producto durante su ciclo de vida (Büyüközkan et al, 2004; Osorio et al, 2013).

Los autores Cheng (2007); Escobar et al (2005), recomiendan seguir las siguientes etapas para un correcto despliegue de la Casa de la Calidad:

- Etapa1. Identificación de la Voz del Cliente (VoC): Al aplicar las encuestas se extrae e identifica las necesidades y expectativas del cliente. Estos resultados significan los "Qués"

- Etapa 2. Asignación del grado de importancia a los "Qués": Se asigna el grado de importancia a los "Qués" con la escala: 3 para "poco 
importante", 4 "importante" y 5 "muy importante".

- Etapa 3. Establecimientos de las Características de Calidad "Cómos": Se elabora una lista de los "Cómos" necesarios para resolver los "Qués".

- Etapa 4. Evaluación competitiva de los "Qués": Se asigna valores en la evaluación competitiva de los "Qués" con escala del 1 al 5 (Pésimo, Malo, Regular, Bueno, Óptimo) para esto se utiliza lo recopilado en las encuestas.

- Etapa 5. Establecimiento del Plan de Mejora: Se establece el plan de mejora de cada ítem de calidad exigida, utilizando las informaciones anteriores, de modo que el producto sea competitivo.

- $\quad$ Etapa 6. Índice de Mejora (IM): Se calcula el índice de mejora dividiendo el valor del plan de mejora entre la puntación de la evaluación competitiva por cada ítem de calidad exigida.

- Etapa 7. Definición del Argumento de Ventas (AV): Se utiliza valores de 1.5 para Venta Especial y 1.2 para Venta Común.

- Etapa 8. Cálculo del Peso Absoluto: Para esta etapa se multiplicó el "Grado de Importancia" por el "Índice de Mejora" por el "Argumento de Ventas" (GI x IM x AV), cada ítem de calidad exigida.

- $\quad$ Etapa 9. Cálculo del Peso Relativo: Se calcula el valor del Peso Absoluto de la etapa anterior en valor porcentual.

- Etapa 10. Desarrollo de la Matriz de Confrontaciones entre los "Qués" vs "Cómos": En esta etapa se valora la influencia que tienen los "Qués" con los "Cómos", para esto se adopta una escala de relación con valores de $1-3-9$, Débil, Medio y Fuerte respectivamente. Los mismos que se multiplican por el valor obtenido en la etapa 8 en ordenadamente.

- Etapa 11. Análisis de los "Cómos": Se estudia la relación "Cómos" Vs "Cómos", para calificar estas relaciones se usan los símbolos: "PF" = Relación Positiva Fuerte; "P" = Relación Positiva; "NF" = Relación Negativa Fuerte; "N" = Relación Negativa.

- Etapa 12. Cálculo de la Puntuación Absoluta: En esta etapa se suma los valores obtenidos en la etapa 10.

- Etapa 13. Cálculo de la Puntuación Relativa: Se calcula la puntuación relativa de los resultados de la etapa anterior en valor porcentual.

- Etapa 14. Establecimiento de la Calidad Proyectada: Analizando la matriz de confrontamiento y demás etapas se establece los valores de calidad proyectada.

- Etapa 15. Interpretación de la Casa de la Calidad: Como última etapa se realiza un análisis holístico de la casa de la calidad.

\section{Despliegue de la Función de Calidad en la cafetería Black Mouth.}

Para el desarrollo de las etapas mencionadas se contó con la participación de tres representantes de la cafetería (gerente, administrador de operaciones y personal de atención al cliente) y la colaboración de un panel de expertos (constituido por tres especialistas en calidad). Pues como afirman Gutiérrez et al (2014) para la aplicación de QFD se demanda el conocimiento a detalle de cada uno de los procesos de producción de bienes y servicios de manera tal que se logre la asignación de prioridades y 
Satisfacción del cliente: Estudio desde las metodologías de calidad. Caso Black Mouth Pablo Alfredo, Rituay Trujillo; Adriano Rogerio, Bruno Tech; Renata Lima, Zuccherelli de Oliveira y Jonathan Alberto Campos Trigoso

objetivos correctamente.

Los datos obtenidos en las encuestas fueron sistematizados en la matriz de la calidad, como se muestra en la figura 1.

Figura 1.

House of Quality - (HOQ)

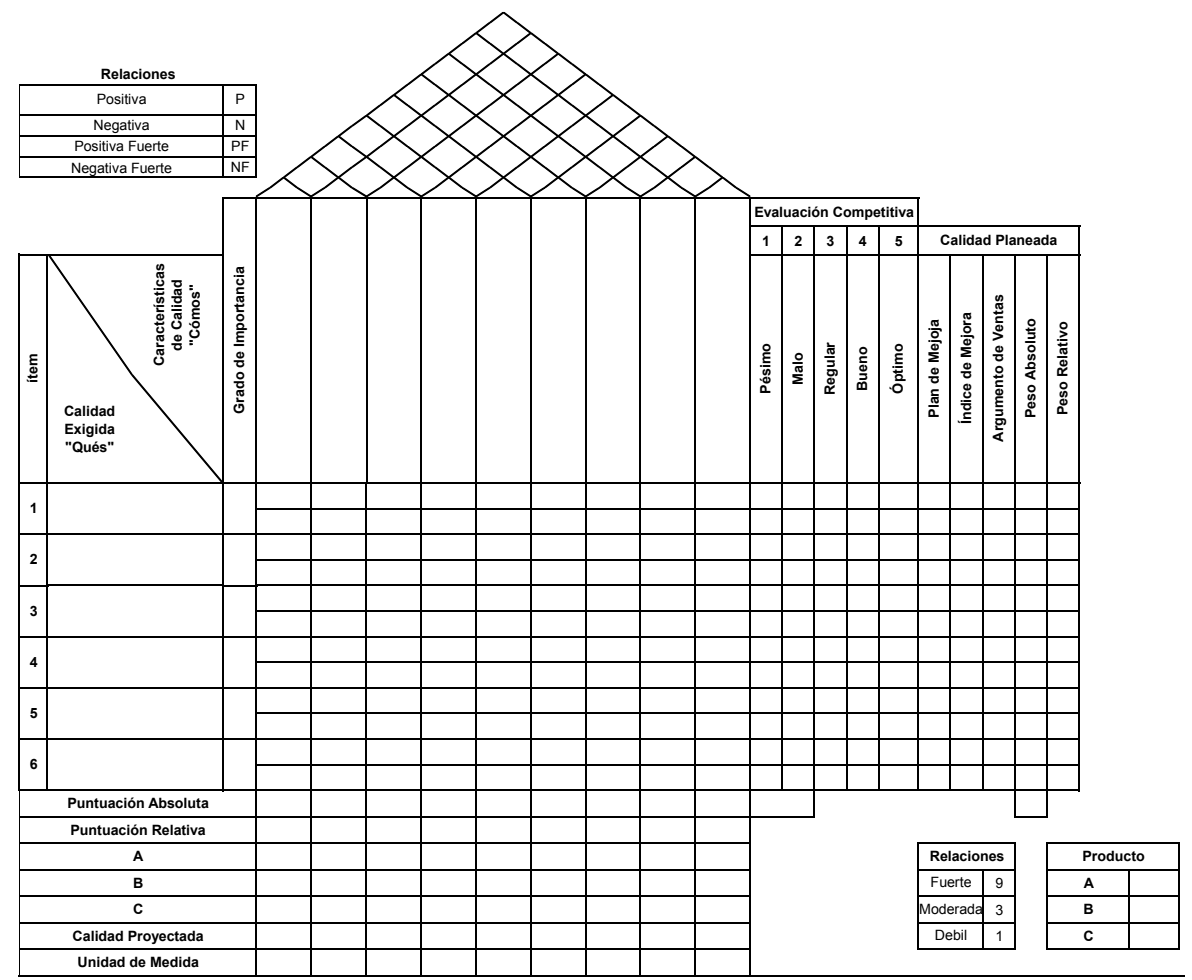

Fuente: Adaptado de Hauser \& Clausing (1988)

\section{Determinación del nivel de satisfacción del cliente}

Con respecto al nivel de satisfacción del cliente, del total de encuestados se aprecia que el $6.6 \%$ lo califica como malo, un $15.8 \%$ como regular, un $25.1 \%$ como bueno y un 52.5 \% como óptimo (Gráfico 1) 
pp. $1290-1303$

Revista Venezolana de Gerencia, Año 24 No. 88, 2019

Tabla 1.

Nivel de satisfacción del cliente

\begin{tabular}{lccc}
\hline & Escala & Frecuencia & Porcentaje \\
\hline Malo & 12 & 6,6 \\
Regular & 29 & 15,8 \\
Bueno & & 46 & 25,1 \\
Óptimo & & 96 & 52,5 \\
\hline & Total & $\mathbf{1 8 3}$ & $\mathbf{1 0 0 , 0}$ \\
\hline
\end{tabular}

Fuente: Elaboración propia

Figura 2.

Nivel de satisfacción del cliente

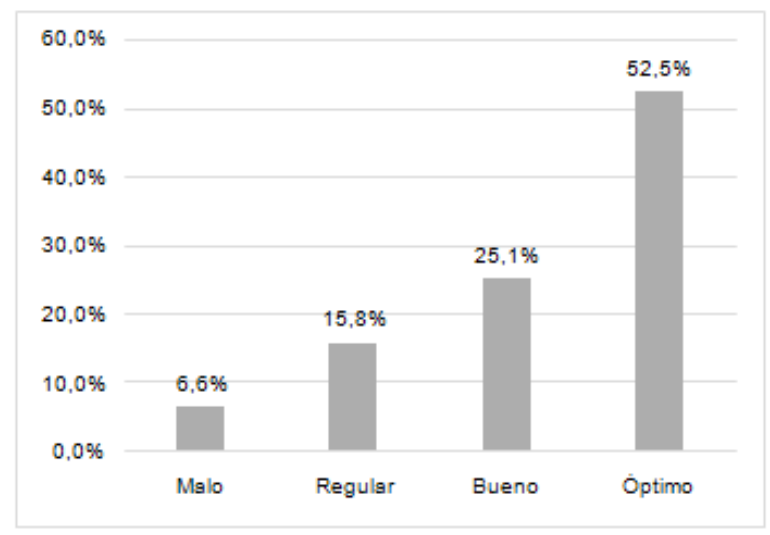

Fuente: Elaboración propia

De acuerdo con los resultados, el nivel de satisfacción del cliente respecto al producto café en taza, es calificado como óptimo por un $52.5 \%$ de los encuestados, teniendo como fundamento las características propias del producto y del ambiente físico de la cafetería referidas a: la intensidad del sabor, temperatura al servir, cantidad servida, presentación agradable, intensidad del olor, precio de venta, atención, ruido, ambiente confortable.

\section{Construcción de la Matriz de la Calidad}

En las siguientes secciones se construye la casa de la calidad para la cafetería Black Mouth

\subsection{Establecimiento de calidad exigida y características de calidad}

Durante la construcción de la matriz de calidad, se establecieron 
las necesidades y expectativas de los clientes a partir del análisis de las encuestas aplicadas en la cafetería Black Mouth.

En el cuadro 1 se observan los seis ítems de evaluación para la calidad exigida por los clientes: sabor, temperatura, cantidad, presentación, olor y precio, a los cuales se asignaron un grado de importancia (poco importante (3), importante (4), y muy importante (5)) como resultado del análisis y consenso del panel de expertos y representantes de la cafetería, estos ítems sirvieron como criterios de evaluación competitiva de los tres tipos de café evaluados.

\begin{tabular}{cccc}
\multicolumn{4}{c}{ Cuadro 1. } \\
Calidad exigida - Los “Qués" \\
\hline Ítem & $\begin{array}{c}\text { Calidad Exigida } \\
\text { "Qués" }\end{array}$ & Valor & Grado de Importancia \\
\hline $\mathbf{1}$ & Sabor & 5 & Muy importante \\
$\mathbf{2}$ & Temperatura & 4 & Importante \\
$\mathbf{3}$ & Cantidad & 3 & Poco importante \\
$\mathbf{4}$ & Presentación & 5 & Muy importante \\
$\mathbf{5}$ & Olor & 5 & Muy importante \\
$\mathbf{6}$ & Precio & 4 & Importante \\
\hline
\end{tabular}

Fuente: Elaboración propia

A partir de los ítems de calidad exigida puntuados se estableció, como se observa en el cuadro 2, las características de calidad con las que se espera dar respuesta a las necesidades y expectativas del cliente respecto al producto y servicio en la cafetería Black Mouth, estos son: intensidad del sabor, temperatura al servir, cantidad servida, presentación agradable, intensidad del olor, precio de venta, atención, ruido y ambiente confortable, todos ellos enfrentados en la etapa de establecimiento de la calidad proyectada y así determinar el grado de relación entre cada una ellas.

Cuadro 2.

Características de calidad - Los "Cómos"

\begin{tabular}{|c|c|c|c|c|c|c|c|c|c|c|}
\hline Ítem & 1 & 2 & 3 & 4 & & 5 & 6 & 7 & 8 & 9 \\
\hline $\begin{array}{c}\text { Características de Calidad } \\
\text { "Cómos" }\end{array}$ & 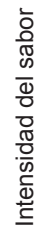 & 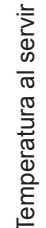 & $\begin{array}{l}\frac{\pi}{0} \\
\sum_{0}^{0} \\
0 \\
0 \\
0 \\
\frac{\pi}{0} \\
.0 \\
0 \\
0 \\
0\end{array}$ & 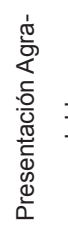 & $\frac{\frac{0}{0}}{\frac{\pi}{0}}$ & $\begin{array}{l}\frac{\overline{0}}{0} \\
\overline{0} \\
\frac{0}{0} \\
\frac{0}{0} \\
\frac{\pi}{0} \\
0 \\
\frac{0}{\Phi} \\
\underline{\underline{D}}\end{array}$ & 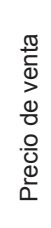 & 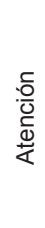 & $\frac{\circ}{\frac{0}{3}}$ & 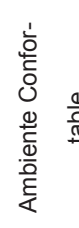 \\
\hline
\end{tabular}

Fuente: Elaboración propia 


\subsection{Desarrollo de la "Evaluación Competitiva" y la "Calidad Planeada"}

La calidad planeada como se observa en el cuadro 3 , se estableció bajo el enfoque de mejora continua y se desplegó con el "Frappe" que presento el menor desempeño al ser evaluado competitivamente con el café americano y café cappuccino.

Cuadro 3.

Evaluación competitiva

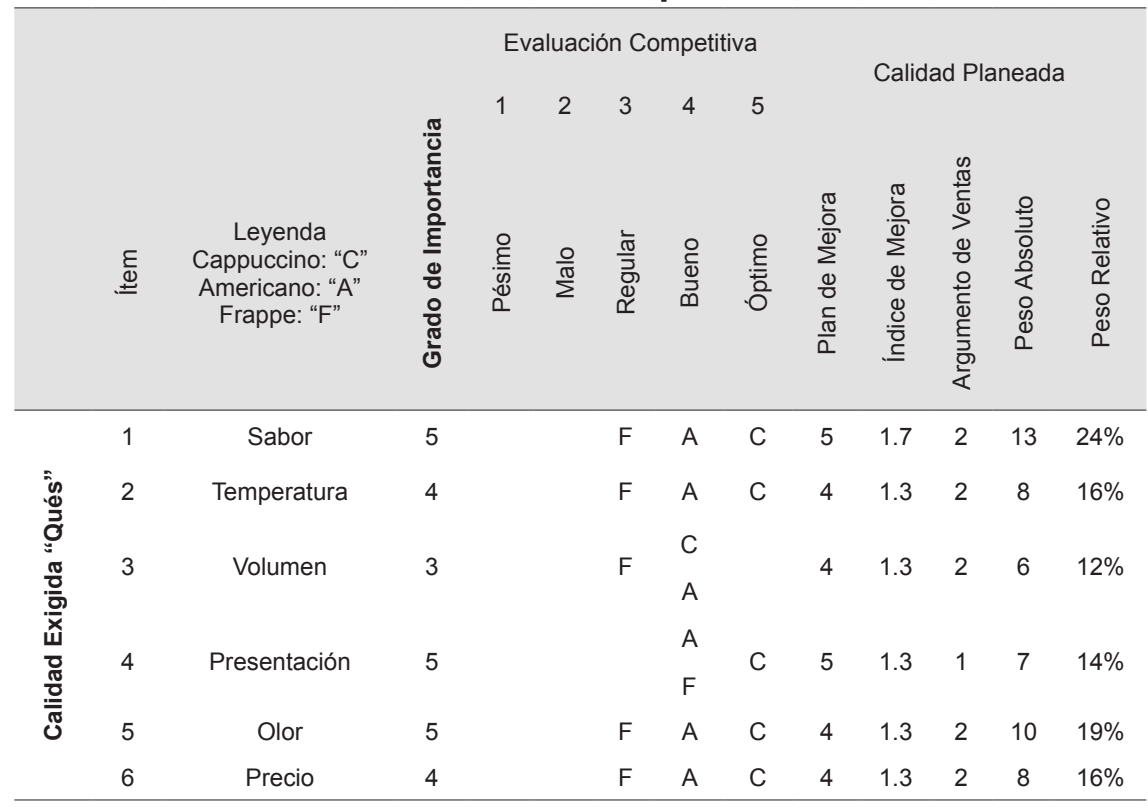

Fuente: Elaboración propia.

\subsection{Matriz de las confrontaciones de los "Cómos" Vs "Cómos"}

A continuación (Cuadro 4) se procedió a la confrontación de las características de calidad para determinar el grado de relación que existe entre cada una de éstas. Se encontró que las relaciones positivas fuertes se presentan en aquellas características directas del producto, tales como: la presentación del café con la intensidad del sabor y la cantidad servida, esto quiere decir que al mejorar alguna de ellas se mejora automáticamente las otras.

Por otro lado, aquellas relaciones negativas se presentaron en características como el ruido y el ambiente confortable, esto quiere decir que al mejorar una, otra puede ser perjudicada y viceversa, finalmente los espacios en blanco muestran que no existe relación 
Satisfacción del cliente: Estudio desde las metodologías de calidad. Caso Black Mouth Pablo Alfredo, Rituay Trujillo; Adriano Rogerio, Bruno Tech; Renata Lima, Zuccherelli de Oliveira y Jonathan Alberto Campos Trigoso

alguna entre las características de calidad, es en estos últimos que resulta necesario tomar acciones con el objetivo de crear algún tipo relación para mejorar la competitividad de la cafetería.

\section{Cuadro 4.}

Matriz de las confrontaciones de los "Cómos" Vs "Cómos"

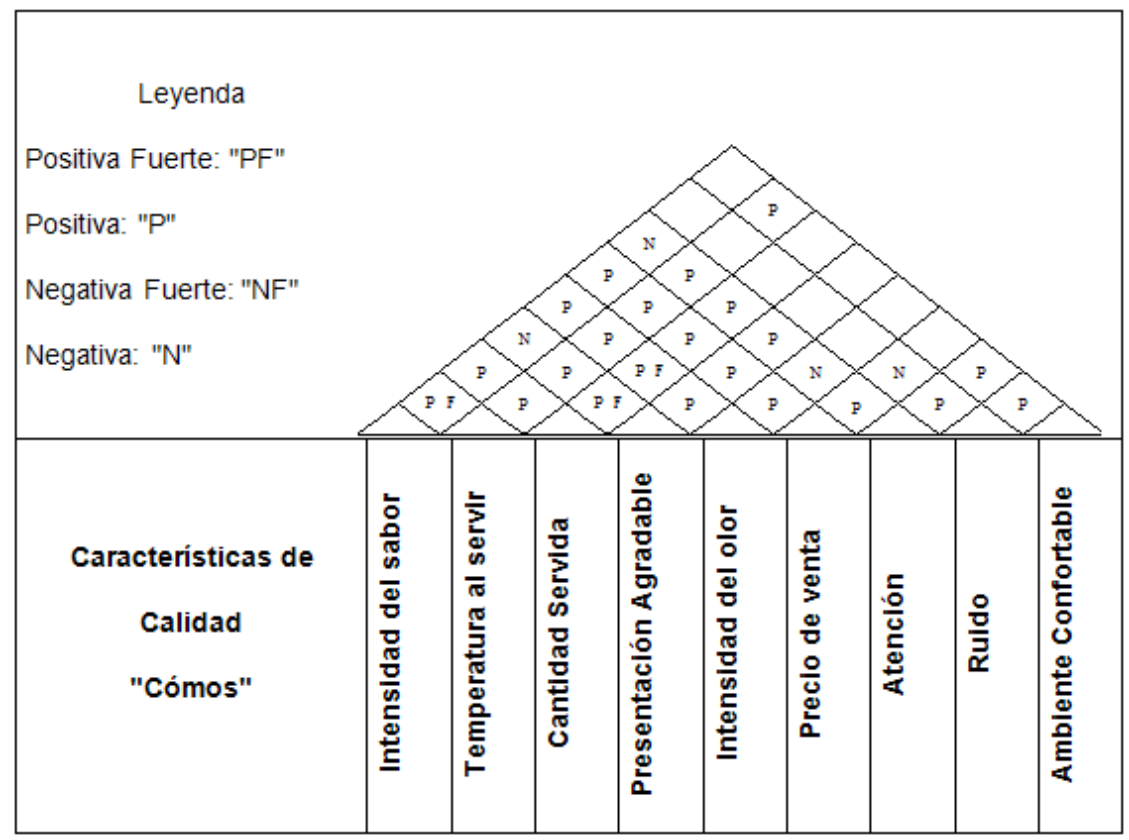

Fuente: Elaboración propia.

\subsection{Matriz de confrontación entre los "Qués" Vs “Cómos}

La matriz de confrontaciones (Cuadro 5) desarrollada por el panel de expertos, con la que se cuantificó la intensidad de la relación entre cada Qué con cada Cómo, utilizando una escala de 1,3 y 9 , asignando 9 en el caso de una relación muy fuerte, 3 para una relación moderada, 1 para una relación débil. 
Cuadro 5.

Matriz de la calidad "Cómos" Vs "Qués"

\begin{tabular}{|c|c|c|c|c|c|c|c|c|c|c|c|}
\hline \multirow{2}{*}{\multicolumn{2}{|c|}{$\begin{array}{c}\text { Confrontaciones } \\
\text { Fuerte: "9" } \\
\text { Moderada: "3" } \\
\text { Débil: "1" }\end{array}$}} & \multirow[b]{2}{*}{$\begin{array}{l}\frac{\pi}{0} \\
\frac{\pi}{\pi} \\
\frac{1}{0} \\
\frac{0}{E} \\
\frac{2}{0} \\
\frac{0}{0} \\
\frac{0}{0} \\
\frac{\pi}{0}\end{array}$} & \multicolumn{9}{|c|}{ Características de Calidad "Cómos" } \\
\hline & & & 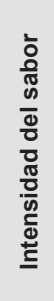 & 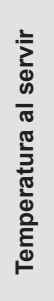 & $\begin{array}{l}\frac{\pi}{0} \\
\frac{0}{2} \\
0 \\
0 \\
0 \\
\frac{0}{0} \\
\frac{\pi}{0} \\
\frac{0}{0} \\
\frac{\pi}{\pi} \\
0\end{array}$ & 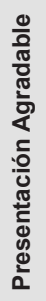 & 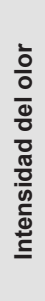 & 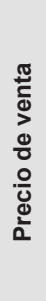 & 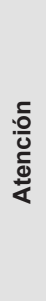 & $\frac{ㅇ ㅡ ㅁ ~}{\frac{0}{3}}$ & 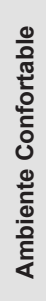 \\
\hline \multirow{12}{*}{ 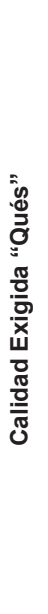 } & \multirow{2}{*}{ Sabor } & \multirow{2}{*}{5} & 113 & 113 & 13 & 13 & 13 & 38 & 13 & 13 & 13 \\
\hline & & & 9 & 9 & 1 & 1 & 9 & 3 & 1 & 1 & 1 \\
\hline & \multirow{2}{*}{ Temperatura } & \multirow{2}{*}{4} & 8 & 24 & 8 & 8 & 72 & 8 & 24 & 8 & 24 \\
\hline & & & 1 & 3 & 1 & 1 & 9 & 1 & 3 & 1 & 3 \\
\hline & \multirow{2}{*}{ Volumen } & \multirow{2}{*}{3} & 54 & 18 & 54 & 54 & 18 & 54 & 18 & 6 & 6 \\
\hline & & & 9 & 3 & 9 & 9 & 3 & 9 & 3 & 1 & 1 \\
\hline & \multirow{2}{*}{ Presentación } & \multirow{2}{*}{5} & 21 & 21 & 21 & 63 & 7 & 63 & 21 & 7 & 63 \\
\hline & & & 3 & 3 & 3 & 9 & 1 & 9 & 3 & 1 & 9 \\
\hline & \multirow{2}{*}{ Olor } & \multirow{2}{*}{5} & 90 & 30 & 30 & 30 & 90 & 30 & 30 & 10 & 30 \\
\hline & & & 9 & 3 & 3 & 3 & 9 & 3 & 3 & 1 & 3 \\
\hline & \multirow{2}{*}{ Precio } & \multirow{2}{*}{4} & 72 & 72 & 72 & 72 & 24 & 72 & 72 & 8 & 72 \\
\hline & & & 9 & 9 & 9 & 9 & 3 & 9 & 9 & 1 & 9 \\
\hline
\end{tabular}

Fuente: Elaboración propia.

\subsection{Puntuación absoluta y relativa de las características de la calidad}

En el cuadro 6, se observa que aspectos como Intensidad del Sabor, Intensidad del olor, Temperatura al servir y precio de venta impactan o están relacionados con varios Qués, por lo que al mejorarse se cumplirían mejor los requerimientos del cliente.

\subsection{Establecimiento de la calidad proyectada}

En el cuadro 7 se observa la calidad proyectada de cada una de las características de calidad de los tres tipos de café: Americano, Cappuccino y Frappe, evaluados a través del uso de la escala de Likert: Pésimo (1), Malo (2), Regular (3), bueno (4) y óptimo (5), Encontrando que aunque la importancia de los Cómo se modificó ligeramente, su orden de prioridad permanece casi igual. 


\section{Cuadro 6.}

Determinación de puntuación absoluta y relativa

\begin{tabular}{|c|c|c|c|c|c|c|c|c|c|}
\hline $\begin{array}{c}\text { Características de Calidad } \\
\text { "Cómos" }\end{array}$ & 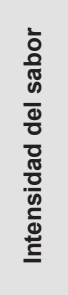 & 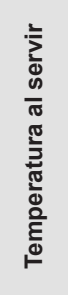 & 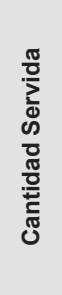 & 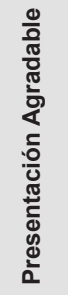 & 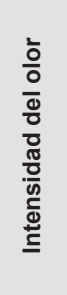 & 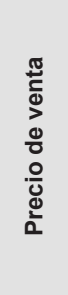 & 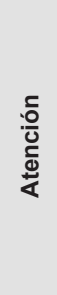 & $\frac{\circ}{\frac{0}{3}}$ & 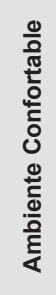 \\
\hline Puntuación Absoluta & 344 & 251 & 198 & 265 & 360 & 240 & 178 & 52 & 208 \\
\hline Puntuación Relativa & $17 \%$ & $13 \%$ & $9 \%$ & $11 \%$ & $15 \%$ & $13 \%$ & $8 \%$ & $2 \%$ & $10 \%$ \\
\hline
\end{tabular}

Fuente: Elaboración propia.

Cuadro 7.

Evaluación competitiva de las características de calidad

\begin{tabular}{|c|c|c|c|c|c|c|c|c|c|}
\hline $\begin{array}{c}\text { Características de } \\
\text { Calidad } \\
\text { "Cómos" }\end{array}$ & 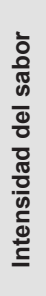 & 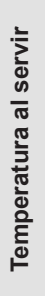 & 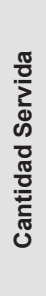 & 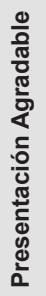 & 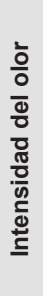 & 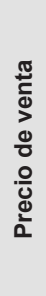 & 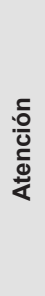 & $\frac{\circ}{\frac{0}{3}}$ & 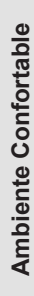 \\
\hline Frappe & 3 & 3 & 3 & 4 & 3 & 3 & 5 & 5 & 5 \\
\hline Americano & 4 & 4 & 4 & 4 & 4 & 4 & 5 & 5 & 5 \\
\hline Cappuccino & 5 & 5 & 4 & 5 & 5 & 5 & 5 & 5 & 5 \\
\hline Calidad Proyectada & 5 & 4 & 4 & 4 & 5 & 4 & 5 & 5 & 5 \\
\hline
\end{tabular}

Fuente: Elaboración propia.

\subsection{Interpretación de la Casa de la Calidad.}

Con lo establecido en la calidad proyectada, a partir de la matriz de confrontaciones entre las características de calidad y la calidad exigida, se confirma lo determinado en la calidad planeada, se tiene que los ítems de características de calidad que obtuvieron una mayor puntuación relativa son las que se priorizaron y proyectaron a mejorar hasta el nivel "óptimo".

Asimismo, de acuerdo con la puntuación relativa se determinaron las características de calidad de mayor interés y atención correspondientes a: intensidad de sabor (17\%), intensidad 
de olor (15\%), temperatura al servir $(13 \%)$ y precio $(13 \%)$, las cuales se deben potenciar prioritariamente para satisfacer las necesidades de los consumidores, estos valores se corroboran al observar los pesos relativos de la calidad planeada $24 \%$,
$19 \%, 16 \%$ y $16 \%$ correspondientes al sabor, olor, temperatura y precio, respectivamente. Sin embargo, no se deben dejar de lado aquellas cualidades exigidas y características que obtuvieron menores valores pues pueden crear conflictos en un futuro (Cuadro 8).

\section{Cuadro 8.}

Casa de la Calidad - HOQ, QFD de la cafetería Black Mouth, 2019.

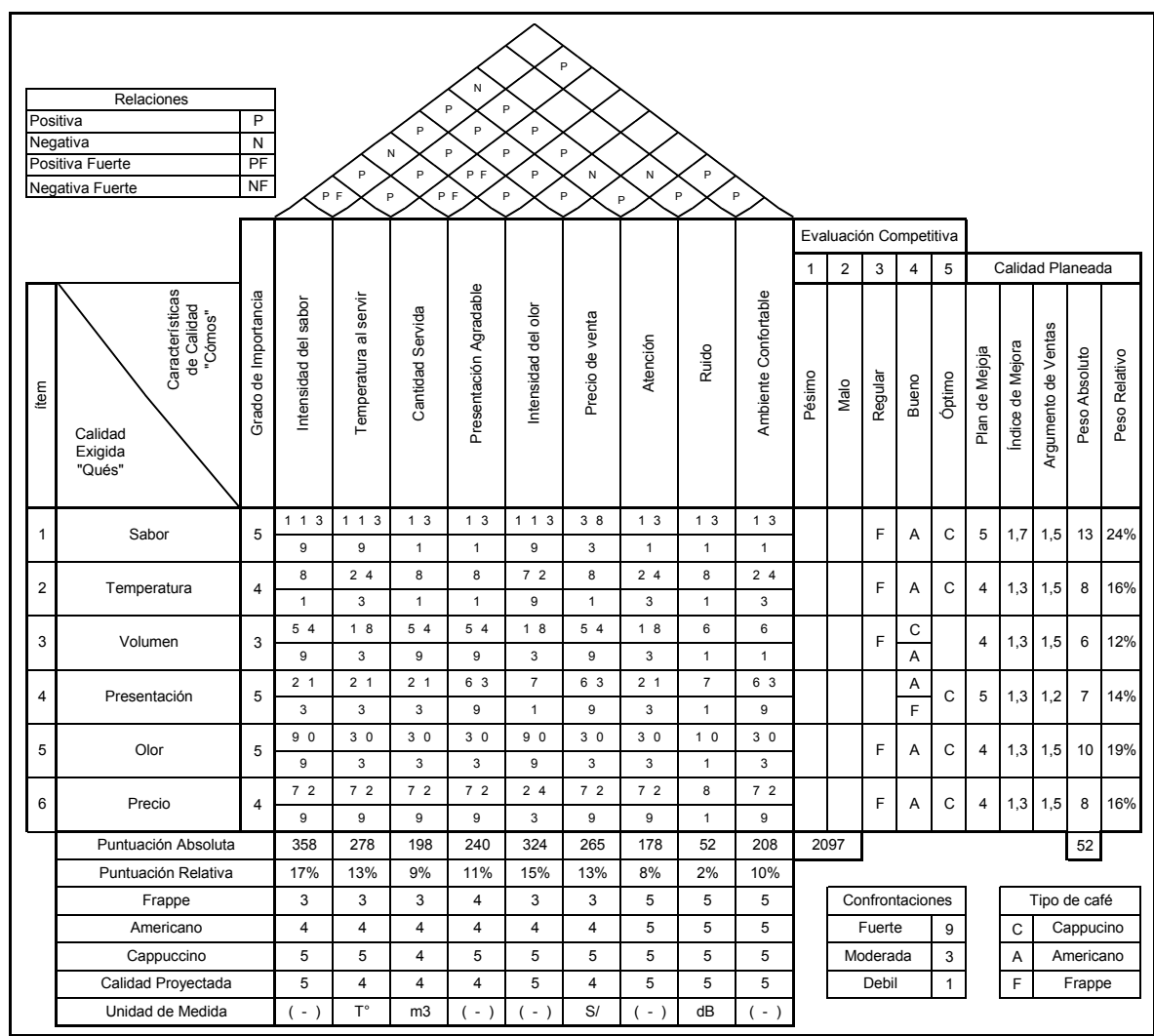

Fuente: Elaboración propia. 
Es así como se identificó las necesidades y expectativas de los clientes con el uso de la metodología QFD coincidiendo con Nieto (2016) quien refiere que el uso de la herramienta QFD ayuda a valorar los requerimientos de los clientes considerados como puntos de mejora a seguir en la organización para desarrollar mejoras del producto, enfocadas en cumplir con las expectativas de los clientes e incrementar las ventas. De igual manera se coincide con (Dias et al, 2015) quienes concluyeron que la metodología QFD ayudo a identificar los requisitos que los clientes desean.

Del mismo modo, se establecieron las características de calidad a partir de los ítems de calidad exigida por los consumidores, coincidiendo con Llontop (2011) quien afirma que el uso de la metodología QFD permite integrar las necesidades del cliente como principal input para el desarrollo de nuevas líneas de productos e impulsar la competitividad de los ya existentes, a través del establecimiento de características de calidad que permiten satisfacer las necesidades de los clientes.

\section{Conclusiones}

Con el desarrollo de la investigación se pudo determinar el nivel de satisfacción del cliente respecto al café en taza en la cafetería Black Mouth de la ciudad de Chachapoyas - 2019 con el análisis de las encuestas aplicadas y el desarrollo de la evaluación competitiva de los productos (café cappuccino, café americano, y frappe) obteniendo como resultado una calificación promedio de satisfacción del cliente óptima.

Con el desarrollo de la matriz de la calidad, principal herramienta del QFD, se identificó la voz del cliente (necesidades y expectativas) que luego fue evaluada competitivamente para determinar aquel producto que presenta un menor desempeño, siendo este el frappe, además permitió identificar los ítems de calidad exigida que tienen mayor representatividad: sabor (24\%), olor (19 $\%)$, temperatura (16\%) y precio $(16 \%)$.

También se definió las características de calidad y la calidad proyectada hacia una valoración óptima, intensidad del sabor (17\%), intensidad del olor (15\%), temperatura al servir (13\%) y precio $(13 \%)$, es sobre éstas que el equipo encargado del desenvolvimiento del producto debe concentrar sus esfuerzos para atender y superar las exigencias de los consumidores.

\section{Referencias Bibliográficas}

Abdul-Rahman, Houssam \& Berawi, Mohammed (2002), " Power quality system," a new system of quality management for globalization: Towards innovation and competitive advantages. Quality Assurance, 9(1), 5-30.

Büyüközkan, Gülçin; Ertay, Tijen; Kahraman, Cengiz \& Ruan, Da, (2004). Determining the importance weights for the design requirements in the house of quality using the fuzzy analytic network approach. International Journal of Intelligent Systems, 19(5), 443-461.

Cheng, Lin (2007), QFD: desdobramento da função qualidade na gestão de desenvolvimento de produtos. Blücher.

Dias, Ananias; Andrade, Carlos, Otávio, José, \& Milan, Marcos (2015). Desdobramento da Função Qualidade (QFD) na Avaliação da Qualidade do Carvão Vegetal Utilizado para Cocção de Alimentos. Floresta e Ambiente, 262-270. 
Escobar, Erika; Rodríguez, Carlos \& Velasco, Óscar (2005), Despliegue de Función de Calidad (QFD) apoyado mediante técnicas difusas: Caso protésis mioeléctrica de mano. Ingeniería e Investigación, 25(2), 4-14.

Evans, James \& Lindsay, William (2015), Administración y control de la calidad. México: Cengage Learning Editores S.A.

Gutiérrez, Humberto; Gutiérrez, Porfirio; Garibay, Cecilia, \& Díaz, Lizbeth (2014), Análisis multivariado y QFD como herramientas para escuchar la voz del cliente y mejorar la calidad del servicio. Ingeniare. Revista chilena de ingeniería, 22(1), 62-73.

Hauser, John \& Clausing, Don (1988), La casa de calidad. Sloan Management Review, 63-73.

Kazemzadeh, Reza; Behzadian, Majid; Aghdasi, Mohammad; \& Albadvi, Amir (2009), Integration of marketing research techniques into house of quality and product family design. The International Journal of Advanced Manufacturing Technology, 41(9-10), 1019.

Llontop, Juan (2011). Mejora en el proceso de desarrollo de nuevas línea de productos basado en la metodología QFD asegurando la calidad del producto a lo largo de su ciclo de vida. Lima, Perú: Universidad Peruana de Ciencias Aplicadas - UPC.

Lorenzo, Susana; Mira, José; Olarte, Mayerly; Guerrero, Johann \& Moyano, Silvia (2004), Análisis matricial de la voz del cliente: QFD aplicado a la gestión sanitaria. Gaceta Sanitaria, 18(6), 464-471.
Mejías, Agustín, \& Manrique, Sergey (2011), Dimensiones de la satisfacción de clientes bancarios universitarios: una aproximación mediante el análisis de factores. Ingeniería industrial, 32(1), 43-47.

Moubachir, Younes \& Bouami, Driss. (2015). A new approach for the transition between QFD phases. Procedia CIRP, 26, 82-86.

Nieto, Ángela (2016), Aplicación de la metodología QFD como mejora de la calidad del servicio en una empresa de alimentacion en la ciudad de Arequipa. Arequipa: Repositorio de Tesis, USCM.

Osorio, Juan; Arango, Diana \& Ruales, Christian (2013), Selección de proveedores usando el despliegue de la función de calidad difusa (supplier selection using fuzzy quality function deployment). Revista EIA, 8(15), 7383.

Proyecto Café y Clima (2017). "Estudio de Mercado del Café Peruano". Proyecto ejecutado por la Cámara Peruana de Café y Cacao, Solidaridad y Plataforma SCAN. Lima.

Tavira, Enrique \& Estrada, Elsa (2015), Marketing relacional: valor, satisfacción, lealtad y retención del cliente. Análisis y reflexión teórica. Ciencia y sociedad, 40(2), 307-340.

Zhang, Xiufen; Zhang, Shuyou; Zhang, Lichun; Xue, Junfang; Sa, Rina, \& Liu, Hai. (2019). Identification of product's design characteristics for remanufacturing using failure modes feedback and quality function deployment. Journal of Cleaner Production, 239, 117967.

- Esta obra está bajo una licencia de Creative Commons Reconocimiento-NoComercialCompartirlgual 3.0 Unported.

http://creativecommons.org/licenses/by-nc-sa/3.0/deed.es_ES 